\title{
Total Vertebrectomy for Non-Small Cell Lung Cancer
}

\author{
Taiji Kuwata $^{a}$ Hidetaka Uramoto $^{a}$ Hajime Ohtomo ${ }^{b}$ \\ Eiichiro Nakamura ${ }^{\mathrm{b}}$ Fumihiro Tanaka ${ }^{\mathrm{a}}$ \\ ${ }^{a}$ Second Department of Surgery and ${ }^{b}$ Department of Orthopedic Surgery, \\ School of Medicine, University of Occupational and Environmental Health, \\ Kitakyushu, Japan
}

\section{Key Words}

Lung cancer $\cdot$ T4 tumor - Invasion of the thoracic vertebra

\begin{abstract}
We present a case who had left upper lobectomy with total vertebrectomy after arterial embolization in preparation for intraoperative bleeding. A 35-year-old man complained of left back pain. Chest $\mathrm{CT}$ revealed a tumor in $\mathrm{S} 1+2$ of the left lung, invading the third thoracic vertebra. As no nodal or distant metastasis was detected, we performed left upper lobectomy and lymph node dissection (ND2a-2) after embolization of the vessels feeding the tumor in order to reduce intraoperative bleeding. In addition, the team of orthopedics performed en bloc resection of the third thoracic vertebra and parts of the left third and fourth ribs. Histological examination of the tumor revealed pleomorphic carcinoma (pT4NOMO, stage IIIA).
\end{abstract}

\section{Introduction}

Among stage IIIA non-small cell lung cancer patients, T4N0-1 cases can be good candidates for surgery and have a relatively favorable postoperative prognosis [1, 2]. However, most T4 cases with thoracic vertebral invasion may not be operated as complete resection with vertebrectomy is challenging [2].

We report the case of a male patient with primary lung pleomorphic carcinoma invading the thoracic vertebra who underwent complete resection with en bloc total vertebrectomy after embolization of the tumor-feeding vessels. 


\section{Case Presentation}

A 35-year-old Japanese man presented with a 6-month history of left back pain. Chest X-ray and CT revealed that a tumor $(43 \times 34 \mathrm{~mm})$ in $\mathrm{S} 1+2$ of the left lung had invaded the third thoracic vertebra (fig. 1a, b). An MRI revealed that the tumor had invaded the third thoracic vertebra and the third rib (fig. 1c). As no nodal or distant metastasis was identified by whole-body CT and bone scintigram, we planned a primary surgery with a clinical diagnosis of lung cancer invading thoracic vertebrae (cT4N0M0, stage IIIA).

To prevent massive bleeding during resection of the tumor with vertebral invasion, arterial embolization was performed prior to surgery, and the first, second, and third intercostal arteries feeding the tumor (fig. $2 \mathrm{a}, \mathrm{b}$ ) were embolized with gelform particles. We performed a complete resection consisting of left upper lobe lobectomy and lymph node resection (ND2a-2), partial resection of the second and third ribs, and total en bloc spondylectomy of the third thoracic vertebra.

Pathological examination showed that all resection margins were free from malignant cells, and the final pathological diagnosis was pleomorphic carcinoma of the lung (pT4N0M0, stage IIIA). We conducted 3 cycles of adjuvant chemotherapy (cisplatin, $80 \mathrm{mg} / \mathrm{m}^{2}$; docetaxel, $60 \mathrm{mg} / \mathrm{m}^{2}$ ) [3] followed by oral administration of S-1 $[4,5]$ and radiation to the primary tumor field. The patient is alive at 14 months after surgery and without any evidence of tumor recurrence.

\section{Discussion}

Patients with primary lung cancer invading the vertebral column are rarely operated $[6,7]$ as the postoperative survival is poor (5-year survival rates, 16-20\%) [6, 8]. However, when complete resection is achieved, favorable prognosis might be expected for such patients $[6,7]$. Thus, complete resection with vertebrectomy, especially total vertebrectomy, is challenging in the field of lung cancer surgery. In the present case, we adopted surgical treatment for the following reasons: (1) no nodal or distant metastasis was identified by whole-body CT and bone scintigram, and (2) complete en bloc tumor resection can be expected by total vertebrectomy of the third thoracic vertebra. Prior to surgery, we performed embolization of the tumor-feeding arteries to prevent possible uncontrollable bleeding during vertebretcomy.

We performed surgery first because complete resection is expected by this technique without preoperative induction therapy. In addition, life-threatening or extremely unpleasant spinal cord invasion and/or meningitis may occur when chemoradiation therapy does not result in tumor shrinkage. Accordingly, we conducted adjuvant chemotherapy and radiation after surgery. As summarized, a careful preoperative evaluation is essential to determine surgical indication, surgical approach, and sequence of multimodality therapy for T4 tumor with vertebral invasion.

\section{Acknowledgements}

We thank Dr. Norihiro Ohnari and Dr. Yu Murakami for their cooperation with embolization of the tumor-feeding arteries. 

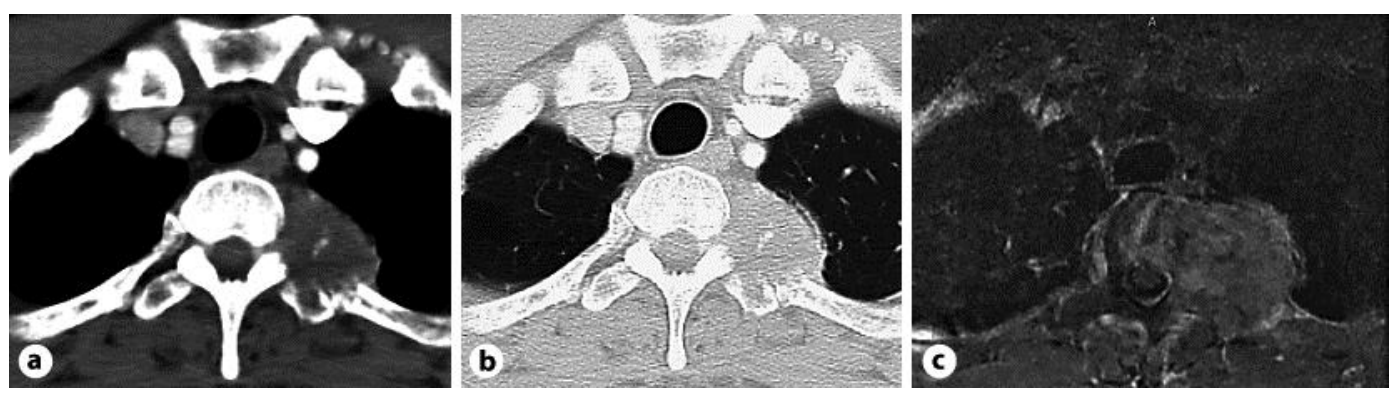

Fig. 1. a, b CT reveals a mass in S1+2 of the left lung, invading the third thoracic vertebra $(84 \times 43$ $\mathrm{mm}$ ). c MRI shows that the mass destroyed the left side of the third thoracic vertebra as it adheres strongly to the left side of the third thoracic vertebra.
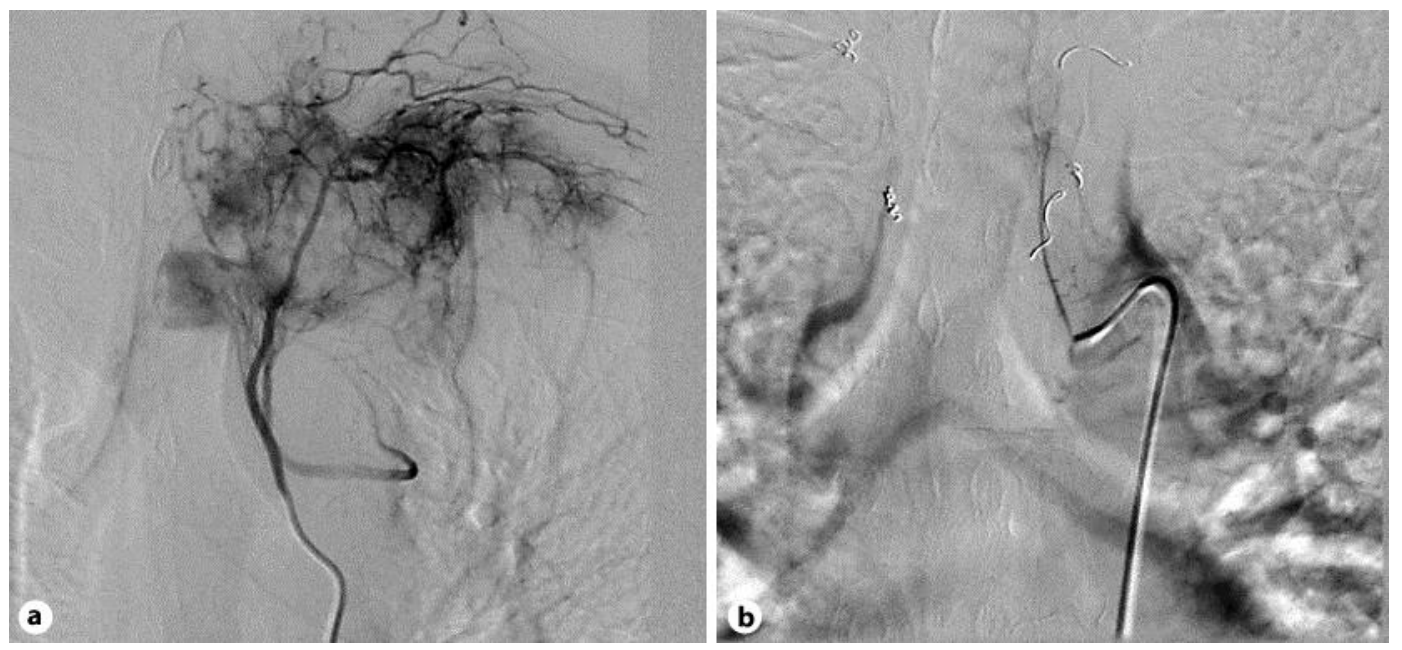

Fig. 2. a Angiography shows that the vessels feeding the tumor were the first, second, and third intercostal arteries. $\mathbf{b}$ The vessels were embolized with gelform particles. Moreover, the first and second left intercostal artery branch to the vertebra was set in vortex coils and, in order to mark up, the right intercostal artery was set in vortex coils. 


\section{References}

1 Chambers A, Routledge T, Billè A, Scarci M: Does surgery have a role in T4N0 and T4N1 lung cancer? Interact Cardiovasc Thorac Surg 2010;11:473-479.

-2 Roy MS, Donington JS: Management of locally advanced non small cell lung cancer from a surgical perspective. Curr Treat Options Oncol 2007;8:1-14.

-3 Kubota K, Kunitoh H, Seto T, Shimada N, Tsuboi M, Okamoto H, Masuda N, Maruyama R, Shibuya M, Watanabe K: A randomized phase II trial of adjuvant chemotherapy with docetaxel (DOC) plus cisplatin (CIS) versus paclitaxel (PAC) plus carboplatin (CAR) in patients with completely resected non-small cell lung cancer (NSCLC): safety and feasibility data from trial TORG 0503. J Clin Oncol 2009;27:15s(abstr 7561).

4 Sakuramoto S, Sakamoto M, Yamaguchi T, Kinoshita T, Fujii M, Nashimoto A, Furukawa H, Nakajima T, Ohashi Y, Imamura H, Higashino M, Yamamura Y, Kurita A, Arai K: Adjuvant chemotherapy for gastric cancer with S-1, an oral fluoropyrimidine. N Engl J Med 2007;357:1810-1820.

-5 Kawahara M, Furuse K, Segawa Y, Yoshimori K, Matsui K, Kudoh S, Hasegawa K, Niitani H: Phase II study of S-1, a novel oral fluorouracil, in advanced non-small-cell lung cancer. Br J Cancer 2001;85:939-943.

-6 Fadel E, Missenard G, Chapelier A, Mussot S, Leroy-Ladurie F, Cerrina J, Dartevelle P: En bloc resection of non-small cell lung cancer invading the thoracic inlet and intervertebral foramina. J Thorac Cardiovasc Surg 2002;123:676-685.

7 Gandhi S, Walsh GL, Komaki R, Gokasian ZL, Nesbitt JC, Putnam JB Jr, Roth JA, Merriman KW, McCutcheon IE, Munden RF, Swisher SG: A multidisciplinary surgical approach to superior sulcus tumors with vertebral invasion. Ann Thorac Surg 1999;68:1778-1784.

-8 Pitz CC, Brutel de la Riviere A, van Swieten HA, Wastermann CJ, Lammers JW, van den Bosch JM: Results of surgical treatment of T4 non-small cell lung cancer. Eur J Cardiothorac Surg 2003;24:1013-1018. 